\title{
Measurement of Mutual Interference Sound of Columnar Objects in Air Flow
}

\author{
Shohji Hamada ${ }^{1}$, Yoshifumi Yokoi ${ }^{2}$ \\ ${ }^{I}$ Graduate student of Mechanical Engineering, National Defense Academy, Hashirimizu 1-10-20, Yokosuka-shi, Kanagawa 239-8686, \\ Japan \\ ${ }^{2}$ Department of Mechanical Engineering, National Defense Academy, Hashirimizu 1-10-20, Yokosuka-shi, Kanagawa, 239-8686, Japan
}

\begin{abstract}
When a columnar object is put in a flow, Karman vortex is formed, and aerodynamic noise is generated. It is known that when multiple columnar objects are put in a flow, the flow state becomes complex. This can be known by flow visualization experiment. On other hands, there are few researches on flow sound in the case of mutual interference, that it is not as far as the authors know. Measurement of flow sound is performed using a microphone. Therefore, it is necessary to confirm the sound of the interference flow field reaches the microphone outside the flow field without changing. In this research, experiments were measured to confirm flow sound transmits to a microphone placed outside the flow field without changing. Based on the results, the aerodynamic noise measurement from the columnar object was performed using a low noise wind tunnel experimental apparatus. As a result, it was obtained that some findings on the sound of flow in mutual interference flow field
\end{abstract}

\section{Introduction}

When a columnar object is put in a flow, a Karman vortex is formed. This phenomenon is a well-known phenomenon in fluid dynamics. Aerodynamic noise is generated by forming the Karman vortex. This matter is also well known phenomenon. There are several flow visualization experiments that when multiple columnar objects are placed in the flow, the flow behind the multiple columnar objects becomes complex [1-4]. In addition, vortex shedding characteristics which generated from multiple columnar objects are also investigated by measurement experiment using a hot wire anemometer [5-7]. It is due to the mutual interference of the flow based on the columnar arrangement state that the state of the flow becomes complex. By contrast, there are few researches on "sound of flow" in the case of mutual interference compared with the research investigated about "state of flow", that it is not as far as the authors know. In order to investigate the flow sound, the microphone measurement is performed. It is necessary to confirm that the sound of the field of interference flow transmits to the microphone placed outside the flow field without changing sound quality. In this research, experiments were measured to confirm "sound of flow" transmits to a microphone placed outside the flow field without changing sound quality. Based on the preliminary results, the aerodynamic noise measurement from the columnar object was performed using a low noise wind tunnel experimental apparatus. The main experimental contents are noise measurement in a single columnar object and noise measurement in multiple columnar objects arrangement which is a mutual interference flow field. As a result, some findings about "sound of flow" in mutual interference flow field were obtained.

\section{Experimental apparatus and method}

\subsection{Experimental apparatus}

The experiment apparatus consists of the low noise wind tunnel, the sound source device, the sound measuring device and two kinds of columnar objects (circular and square cylinders). The low noise wind tunnel has an air outlet and an air inlet in the anechoic chamber. The shape of air outlet is regular octagon. (one side of length is 200 $\mathrm{mm}$; opposite side of length is $475 \mathrm{~mm}$ ) The wind tunnel can cause air flow of up to $40 \mathrm{~m} / \mathrm{s}$ and disorder strength at $20 \mathrm{~m} / \mathrm{s}$ is $1 \%$. In this experiment, the air flow range is from $0 \mathrm{~m} / \mathrm{s}$ to $30 \mathrm{~m} / \mathrm{s}$. The sound source device consists of the frequency generator, the integrated amplifier and the frequency counter. The sound measuring device consists of the condenser microphone (1/2 inch), the precision sound level meter and the FFT analyzer. The microphone is attached a wind screen $(\varphi 70$, made of urethane foam). Two kinds of columnar objects are two circular cylinders and two square cylinders. The circular cylinder diameter and the side of the square cylinder are $20 \mathrm{~mm}$, respectively.

\subsection{Experimental method}




\subsubsection{Preliminary experiment}

Four kinds of preliminary experiments were performed to confirm that the sound quality does not change by the airflow. The air flow was passed between the microphone and the sound source, and the sound was measured. Air velocity range was from $0 \mathrm{~m} / \mathrm{s}$ to $30 \mathrm{~m} / \mathrm{s}$. Sound pressure level of the sound source range was from $40 \mathrm{~dB}$ to $80 \mathrm{~dB}$. The frequency of sound source was $440 \mathrm{~Hz}$. The microphone and the sound source were set apart from each opposite by $1000 \mathrm{~mm}$ from the center of wind tunnel outlet. The sound was measured by changing the frequency from the sound source. The placement of the experimental apparatus was the same as in the previous experiment. Air velocity range was from $0 \mathrm{~m} / \mathrm{s}$ to $30 \mathrm{~m} / \mathrm{s}$. Sound pressure level of the sound source was $50 \mathrm{~dB}$. Frequency range was from $130 \mathrm{~Hz}$ to $1960 \mathrm{~Hz}$. These frequencies were set for each musical scale of 4 octaves. A cylinder was placed in the airflow and the sound passed through the turbulent airflow was measured. Air velocity range was from $0 \mathrm{~m} / \mathrm{s}$ to $30 \mathrm{~m} / \mathrm{s}$. The sound pressure level of the sound source was $60 \mathrm{~dB}$. The frequency of the sound source was $440 \mathrm{~Hz}$. The cylinder was placed in the airflow. The microphone and the sound source were installed at the position of $1000 \mathrm{~mm}$ on both sides from the position of the installed cylinder wake flow of $500 \mathrm{~mm}$. The sound source was installed in the airflow and the sound was measured. Air velocity range was from $0 \mathrm{~m} / \mathrm{s}$ to $30 \mathrm{~m} / \mathrm{s}$. The sound pressure level of sound source range was changed from $50 \mathrm{~dB}$ to $70 \mathrm{~dB}$. The frequency was $440 \mathrm{~Hz}$. The sound source was installed in the airflow, and the microphone was installed at $1000 \mathrm{~mm}$ from the sound source.

\subsubsection{Two circular cylinders case}

Two parallel arrangenet circular cylinders were installed in the airflow, and the aerodynamic sound generated by the distance ratio was measured using a microphone installed outside the airflow. A schematic view is shown in Fig 1. Two cylinders were fixed at $500 \mathrm{~mm}$ of the outlet. Fig. 1 (b) showed the installation state of two cylinders. Two cylinders can be changed distance between cylinders by attatchment equiped on the outlet.The diameter of the two cylinders was same $(D=$ $20 \mathrm{~mm})$. The center of two cylinders was set the $(500,0)$. It had been confirmed that two cylinders did not resonate duaring experiment. The microphone was set the $(500$, 1000). The air velocity was set $20 \mathrm{~m} / \mathrm{s}$. Two cylinders were set the distance ratio indicating the characteristic flow $(G / D=0.25,0.75,1.75)[5-7] . G / D$ is called distance ratio, after here. $G$ is the distance between the surface of cylinders. $D$ is diameter. The characteristic flow in the previous research is that when the distance ratio is large, the vortices generated from the two cylinders are the same as the vortexes generated from independent each single cylinders. When the distance ratio decreases, each vortex generated from the two cylinders releases a different vortex. When the distance ratio is small, two cylinders emitt a vortex that the two cylinders are generated from like a single cylinder having twice the diameter.

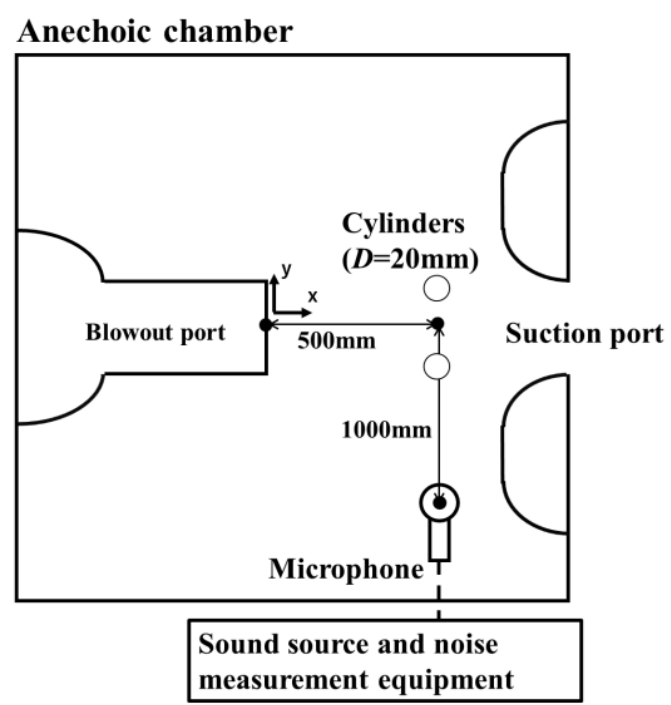

(a)

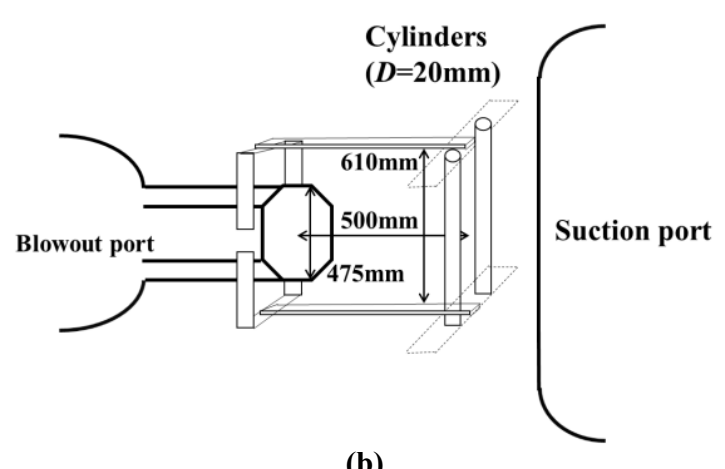

(b)

Figure 1. Experimental apparatus placement in anechoic chamber;(a) Top view, (b) Side view

\subsubsection{Two square cylinders case}

The experiment of using two square cylinders was performed for investigating influence of changing separation point. Because a separation point of circular cylinder is not constant in the airflow. On the other hand, a separation point of square cylinder is constant. Two parallel arrangement square cylinders were installed in the airflow, and the aerodynamic sound generated by the distance ratio was measured using a microphone installed outside the airflow. Two square cylinders were fixed in the rear stream of the outlet as the case of circular cylinders. The diameter of the two cylinders was same $(D$ $=20 \mathrm{~mm})$. The center of two cylinders was set the $(500$, $0)$. The microphone was set the $(500,-1000)$. The air velocity was set $25 \mathrm{~m} / \mathrm{s}$. Two cylinders were set the distance ratio indicating the characteristic flow $(G / D=$ $0.6,1.7,4.9)$. Previous research showed the characteristic flow [3]. When the distance ratio is large, the vortices generated from the two cylinders are the same as the vortexes generated from independent each single cylinders. When the distance ratio decreases, each vortex generated from the two cylinders releases alternating vortexes and twin vortex. When the distance ratio is small, two cylinders emitt alternating vortexes and one side of the vortex. 


\section{Experimental results and discussion}

\subsection{Preliminary experiment results}

From the four preliminary experiments, the following was obtained. The sound does not change its quality even across the airflow. This phenomenon is not dependent on frequency. The same result can be obtained even if the airflow is turbulent. The sound generated in the airflow is transmitted outside the airflow without changing its quality.

\subsection{Two circular cylinders case}

Fig. 2 shows the relationship between vortex shedding frequency and sound pressure level obtained from a microphone measurement experiment. Airflow velocity was $20 \mathrm{~m} / \mathrm{s}$. The Strouhal number was about 0.18. $(\mathrm{St}=f$ $d / U, f$ : vortex shedding frequency, $d$ : cylinder diameter, $U$ : mainstream velocity) This is the same result as the case of using hot wire anemometer experiment. Fig. 3 shows the relationship obtained from two parallel arrangement circular cylinders experiment between vortex shedding frequency and sound pressure level at each distance ratio. In the case of previous study using hot wire anemometer, two frequency peaks can be seen at the distance ratio 0.75 . But in each other distance ratios, there was only one frequency peak. Fig. 4 showed the relationship between the distance ratio and the Strouhal number. At a point of distance ratio 1.75, the present result shows the same Strouhal number with the case of a single cylinder. Other distance ratios were different from those of the previous research. It considered this difference was caused the influence of the background noise, the change of the sound, and the change of the separation point. However, they did not cause the difference. In the Fig. 3, the sound pressure level of the background noise is not large so it could not affect the result. From preliminary experiment results, the sound generated inside the airflow transmits to the outside the airflow with not changed its quality.

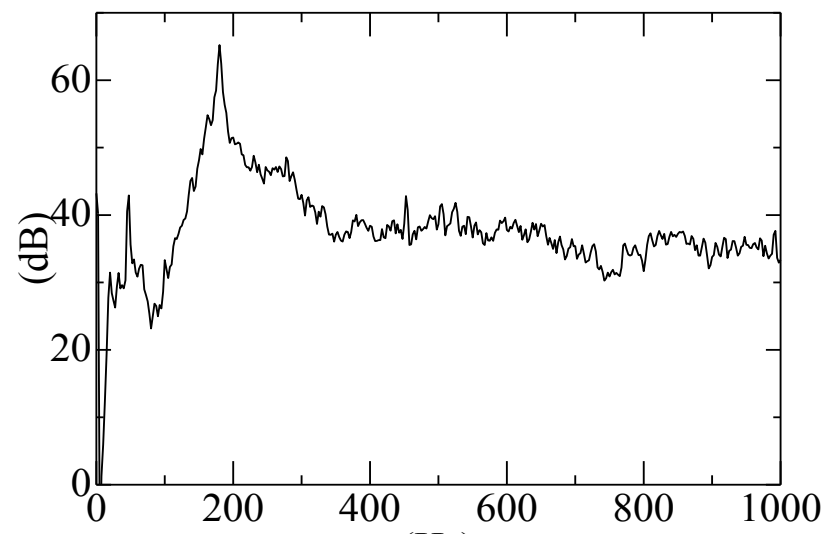

$(\mathrm{Hz})$

Figure 2. Single circular cylinder: Relationship between vortex shedding frequency and sound pressure level

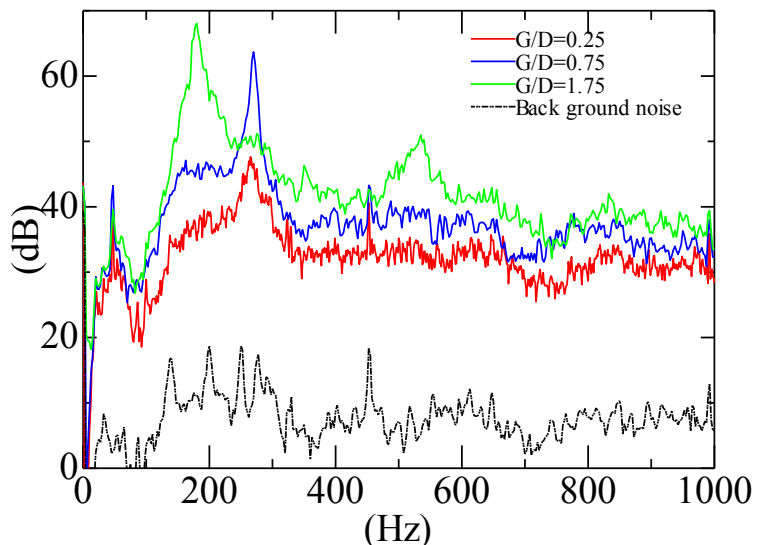

Figure 3. Two parallel circular cylinders: Relationship between vortex shedding frequency and sound pressure level

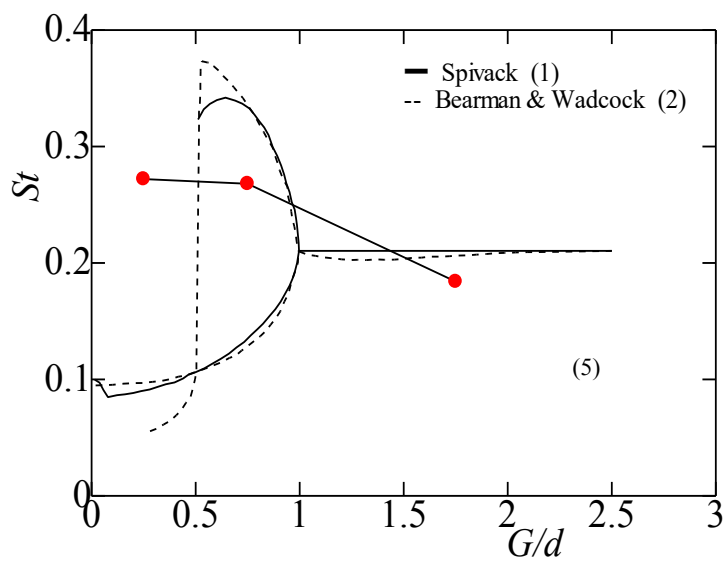

Figure 4. Two parallel circular cylinders: Relationship between distance ratio and Strouhal number

\subsection{Two square cylinders case}

The generated sound from single square cylinder was measured. Airflow velocity was $25 \mathrm{~m} / \mathrm{s}$. Fig. 5 shows the relationship between vortex shedding frequency and sound pressure level obtained from a single cylinder experiment. The Strouhal number was about 0.124 . This is the same result as the result of using hot wire anemometer result. Fig. 6 shows the relationship obtained from this experiment between vortex shedding frequency and sound pressure level at each distance ratio. At distance ratio 1.7 and distance ratio 4.7 , one frequency peak can be seen. At distance ratio 0.6, the frequency peak cannot be seen. Fig. 7 shows the relationship between the distance ratio and the Strouhal number. At a distance ratio 4.9, the result shows the same Strouhal number with the case of a single square cylinder. At distance ratio 1.7 , multiple frequency peaks cannot be seen. At distance ratio 0.6 , there is no frequency peak. This is also different from previous research. Experimental results in the case of a square cylinder and the case of a circular cylinder are same results. That is, the measurement result by the hot wire anemometer is different from the measurement result by the microphone.This result shows that the vortex shedding frequency is different from the frequency of sound of mutual interference; this shows it cannot be trusted to know the condition of objects only by the result of the 
sound. For example, even if two objects are present at any distance ratio, the frequency obtained by sound is one frequency:thus it cannot be not known the condition of objects.

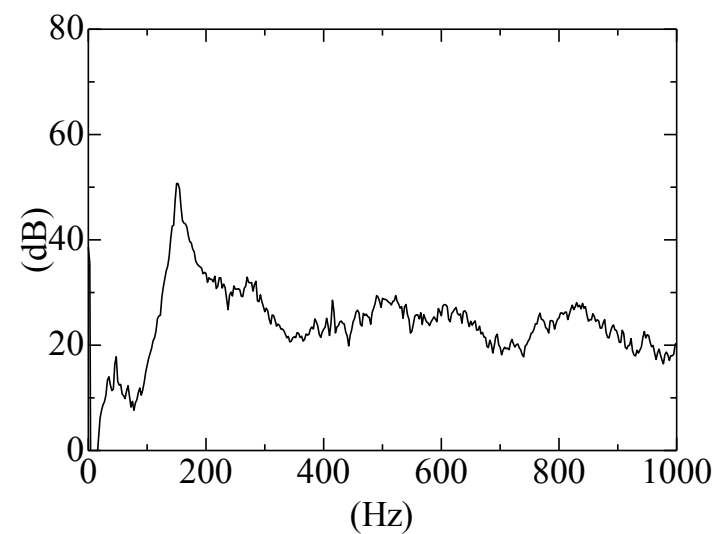

Figure 5. Single square cylinder: Relationship between vortex shedding frequency and sound pressure level

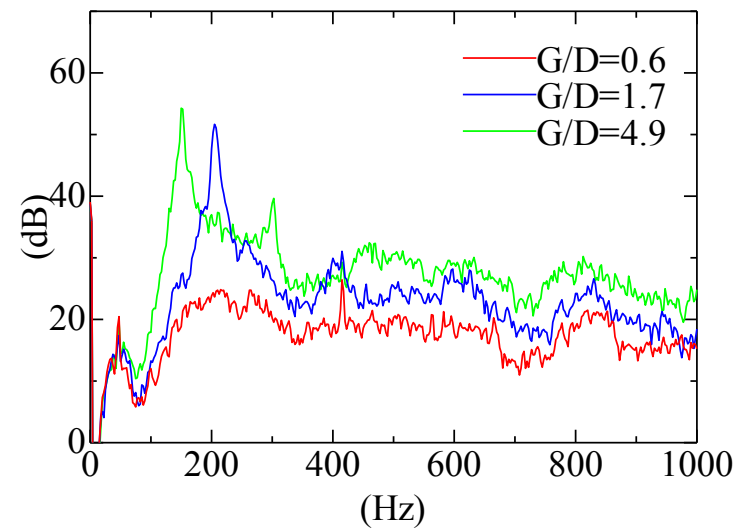

Figure 6. Two parallel square cylinders: Relationship between vortex shedding frequency and sound pressure level

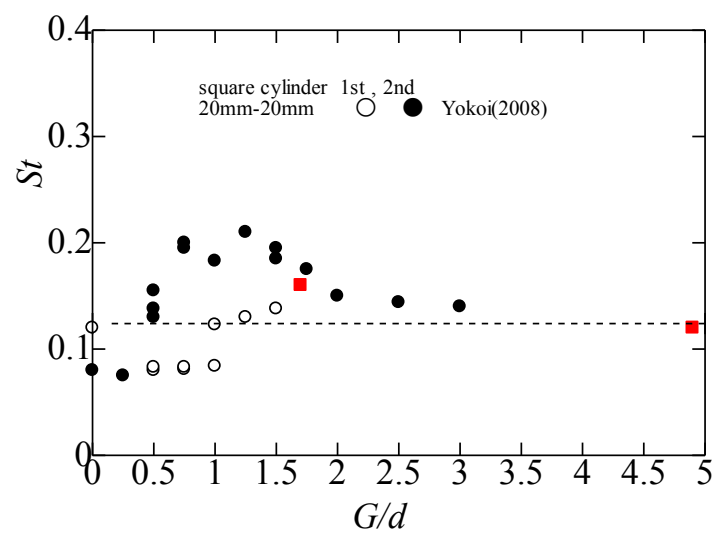

Figure 7. Two parallel square cylinders: Relationship between distance ratio and Strouhal number

\section{Conclusions}

The mutual interference flow from the two columnar objects installed in the airflow was measured by the microphone. The following conclusions were obtained.

(1) The quality of the sound generated in the airflow can be known outside the airflow without changing the quality.

(2) In the case of a single columnar object, it was confirmed that the measurement result of the hot wire anemometer and the measurement result of microphone became same result.

(3) As a result of measuring the sound of mutual interference flow and analyzing the frequency of the sound, it was shown that it is different from the result of the hot wire anemometer.

(4) In the case of square cylinder, also the same result as the conclusion (3) was obtained.

\section{References}

1. Ohumi, K., Imaichi, K. and Tada, E., On Vortex Streets Behind Circular Cylinders in Side-by-Side Arrangement, Journal of the Visualization Society of Japan, Vol. 10, Suppl. No. 1 (1990), pp. 35-40.

2. Ohumi, K., Visualization Experiment and Numerical Calculation on Two Circular Cylinders in Staggered Arrangement, Journal of the Visualization Society of Japan, Vol. 15, Suppl. No. 1 (1995), pp. 217-220.

3. Y Yokoi, K Hirao, The interation Vortex Flow Around a pair of Parallel Arranged Bluff Cylinders. (In the case of stationary setting), Proceedings of the Japan Society of Mechanical Engineers (Part B) 74 vol. 741(2008-5)

4. Moriya, M. at al., Fluctuating Fluid Forces of Two Circular Cylinders in Parallel Arrangement at Close Spacing, Transaction of the Japan Society of Mechanical Engineers, Series B, Vol. 68, No. 676 (2002), pp. 3310-3315.

5. Spivak, H. M., Vortex Frequency and Flow Pattern in the Wake of Two Parrallel Cylinders at Varied Spacings Normal to an Air Stream, Journal Aeronatutical Sciences, Vol. 13 (1946), pp. 289-297.

6. Bearman, P. W. and Wadcock, A. J., The Interaction Between a Pair of Circular Cylinders Normal to a Stream, Journal of Fluid Mechanics, Vol. 61, Part 3 (1973), pp. 499-511.

7. Kobayashi, T., Characteristics of Fluid-Dynamic Forces Acting on Circular or Square Cylinders in Close Proximity, Transaction of the Japan Society of Mechanical Engineers, Vol. 42, No. 357 (1976), pp. 1452-1461 